\title{
Multimedia Service using CSMA/GPS in Vehicular Communication Network
}

\author{
Backhyun $\mathrm{Kim}^{1}$, Yoseop $\mathrm{Woo}^{2}$ and Iksoo $\mathrm{Kim}^{2 *}$ \\ ${ }^{1}$ Faculty of Liberal Education, Incheon National University, \\ ${ }^{2}$ Dept. of Information and Telecommunication Engineering Incheon National \\ University \\ 12-1 Songdo-dong Yeonsuku, Incheon, Korea \\ \{bhkim24,yswoo and iskim\}@incheon.ac.kr
}

\begin{abstract}
This paper ${ }^{1}$ deals with the seamless multimedia service mechanism in vehicle communication network(VCN). The proposed mechanism uses caching/switching/multicasting agent(CSMA), a partial of GPS information and multioasi transmission in VCN network. The CSMA controls some smart access points(SAP) with buffer, and may generate multicast group address for live-video in local-vehicle-network $(L V N)$. And it manages serviced video items, switches multimedia packets to SAPs requesting Gervice, and performs smooth handover for vehicles using table. The SAP generates modified virtual address(IP address) for vehicles that enter the VCN network. The proposed virtual IP address consists of LVN network ID, vehicle ID, GPS information(dving distance) and a driving direction mode bit.

And the CSMA and SAP provide smooth handover using their service tables and a seamless multimedia service. Also, this proposed mechanism may provide promptly multicast service for live-video because multicast mechantsm is performed within the VCN network, and reduce the possibility of cutoff service and packet loss because it uses GPS in-formation.
\end{abstract}

Keywords: V2V, V2I hetwork, Multicast, Multimedia, Handover, GPS

\section{Introduction}

In recent year, the researches in wireless network may classify into two main fields, one is the conventional wireless network and the other is vehicle-based wireless network. Insufficient network resources is the one common critical problem in the conventional wireless network and vehicular wireless communication network(VCN), that is a problem of shortage for network bandwidth. Eventually, extensive researches to provide various multimedia service in the conventional wireless network and vehicle-based wireless network widely being researched all over the world. The two main fields of those are to use more effecti ely network resources and to improve overall efficiency including the excessive load of multimedia server in such networks.[1-3]

And the vehicle-based wireless networks are divided into two groups, maybe one main group is the vehicle-to-infrastructure(V2I) network and the other one is the vehicle-tovehicle(V2V or ad-hoc mode) network $[1,4]$.

\footnotetext{
* Corresponding author

${ }^{1}$ This paper is extended and revised one(Video Service using Smart AP and GPS in Vehicle Network) that was presented at the CES CUBE 2014(Saipan) conference
} 
The vehicle-to-vehicle(V2V or ad-hoc mode) network is equipped processors with various sensors, and generally communicates using multi-hop mode(short range communications) without any infra-structured network elements such as access pointer(AP) and basic service set(BSS). The vehicle-to-infrastructure network is called V2I(vehicle-to-infrastucture) using existing network infrastructure (e.g., AP or RSU). The various service such as intelligent transportation systems, a variety of safety applications, traffic information, maps download and multimedia services(game, MP3 and live-video for sports relay broadcasting) for entertainments are provided in these vehicle-based network in the world over the past few years $[4,5]$.

Providing seamless multimedia services for entertainments in the VCN network, the VCN networks have to solve some critical problems that are cut off connection, reestablishing pathconnection and the loss of packets[2, 5, 6].

This paper presents seamless multimedia service mechanism using caching/switching/multicasting agent(CSMA), some smart APs(or smart RSU road side unit) with buffer and a partial of GPS information(the speed(or position of vehicles) in V2I network. This proposed mechanism can be used for intelligent transportation system(ITS) and traffic alerts for safety if it uses multi-hop mode(vehicularad hoo network. VANET), and let them reserve for future work.

The proposed mechanism solves the drawbacks of reconnection and cutoff in the process of handover, uses effectively network resources guarantees prompt service and reduces the load of server because multicast mechanism for live-video does not performed on the server but the CSMA within VCN network. Also, it adopts the GPS information(the speed/or position of vehicles) to reduce the possibility of packethoss that may have occurred during handover. The proposed mechanism can perform these functions that applying a virtual IP address including a partial GPS inforpration and an adding only network ID number moved to original virtual IP address assigned from the first accessed SAP in VCN network [5, 7].

The rest of this paper is as follows: Section 2 presents the structure of seamless multimedia service mechanism in VCN network, and section 3 deals with the operation mechanism for seamless multimedia service using CSMA/GPS in VCN network. Section 4 and 5 discusses algorithm for the proposed mechanism and the simulation results, respectively. And finally, we discuss our conchusion.

\section{The Structure of Mutimedia Service System in Vehicle Communication Network}

The structure of the proposed multimedia service system using CSMA, a partial GPS information and multicast mechanism in VCN network is shown in Figure 1, and that is just like mentioned in paper 7.

The VCN hetwork that providing seamless multimedia service using the proposed mechanism consists of some local-vehicle-network(LVN). The LVN is composed of a CSMA(caching/switching/multiplexing agent), a few SAPs/or S-RSUs(smart-RSU) and some vehicles that are controlled by the SAP. The structure of the proposed VCN network is very similar to that of the paper 7 .

As shown in Figure 1, the proposed multimedia service system consists of multimedia server, some LVN networks connected through Internet and many driving vehicles in VCN network 


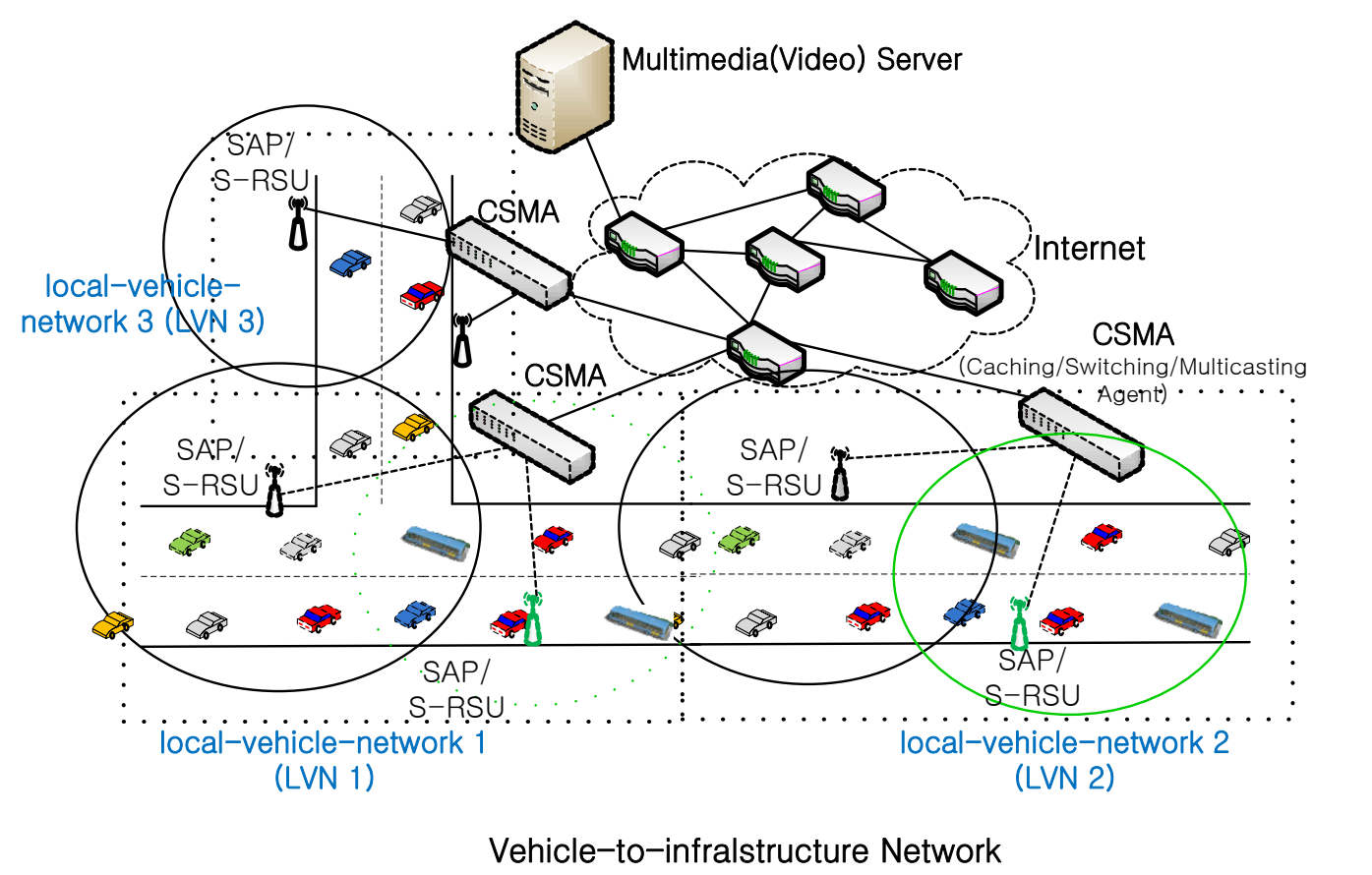

Figure 1. The Structure of the Proposed Multimedia Service System in Vehicle Communication Network(VCN)

\section{The Operation Mechanism for Multimedia Service System using CSMA/GPS in Vehicle Communication Network}

Section 3 deals with the operation of the proposed multimedia service system shown in Figure 1. The VCN networe is made up of some of LVN networks. The SAP/S-RSU in a LVN network assigns a virtual IP address when vehicles request connection or enter into the VCN network. A vitual IP address is composed of two LVN IDs(the 1st accessed LVN network and new (1) moved LVN network), one the 1st accessed-SAP ID, a vehicle ID(assigned by the 1 st accessed SAP) and a vehicle speed field(or driving distance within LVN network).

Figure 2 shows the format of the virtual IP address issued by SAP. The length of virtual IP address is 32-bits long including 2-bit reserved field for future use. As shown in Figure 2, two LVN IDs(8-bit long) Indicate newly moved LVN network ID(4-bit) and the 1st accessed LVN ID(4-bit). The 1st accessed LVN network ID is assigned from a SAP accessed when a vehiclei enters the LVN network in VCN network for the first time, and the moved LVN network ID is newly assigned from a SAP accessed when the vehiclei enters the other LVN from the 1 st LVN. This moved LVN network ID can be changed in accordance with the vehiere's driving. But the 1st LVN network ID never changes in accordance with the vehicle's driving.

The vehicle ID field is 8-bits long, the proper number of vehicles entered VCN network and the vehicle speed field(8-bits long) indicates driving distance within a specific LVN network as a partial GPS information. Another use of this GPS information is to provide smooth handover technique and to reduce the possibility of packet loss during handover. And 2-bit long mode bit indicates the direction of vehicle's driving.

The CSMA performs switching function for multimedia streams transmitted from the server. This means that it switches multimedia streams to SAPs requesting service in a 
specific LVN network[7]. And it has a cache to store multimedia streams and switchingmapping table to indicate that the serviced streams are transmitted from CSMA to SAP of a certain LVN network. In other words, the mapping table indicates the relationship of SAPs and vehicles accessed within LVN network because this relationship can change frequently in accordance with the vehicle's driving. This switching function and the switching-mapping table of CSMA is the basis of smooth handover. Let the detailed explanation for the switching-mapping table is at the back of this section. Also, the CSMA provides multicast transmission mechanism with respect to that a number of vehicles request the same livevideo. Thus, it creates a multicast group for them and generates a multicast group address (MGA), and then sends multicast streams to the vehicles through SAP accessed. And the CSMA transmits live-video streams with a MGA to the SAPs, and then the vehicles in each LVN network join a multicast group with a MGA.

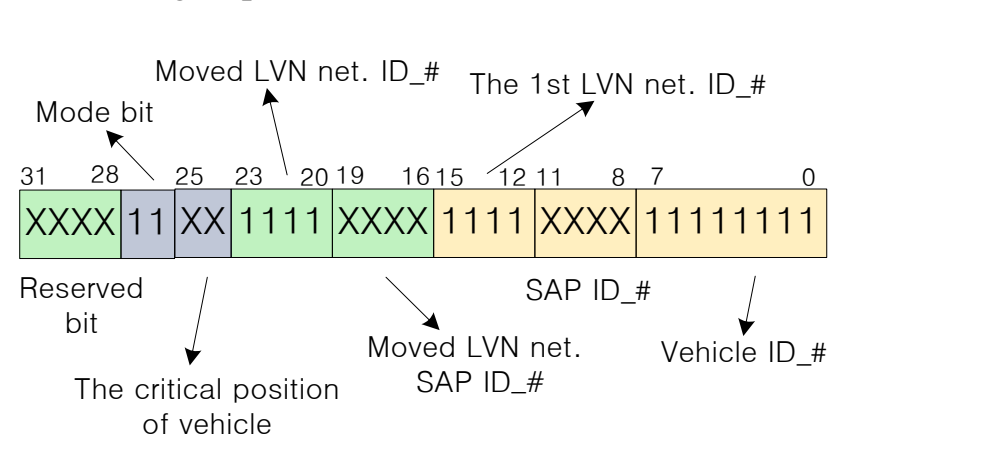

Figure 2. The Format of Virtualip Address

The SAP in LVN network controls vehiclesaccessed within its communication coverage area. It assigns a virtual IP address(Figure 2) to Vehicles when they enter the VCN network for the first time. It never changes the 1st LVN network ID_\#, SAP ID_\# and vehicle ID_\# fields of a virtual IP addres when the vehicle enters other LVN networks. But the SAPs in another LVN networks change only moved LVN network ID field when the vehicle assigned the virtual IP address for the first ime is driving into other LVN networks. Figure 3(a) shows a virtual IP address(including vehrele ID_\#21) issued by SAP ID_\#7 when a vehiclei enters LVN network ID \#5 in VCN network for the first time. And Figure 3(b) shows a virtual IP address changed by SAP ID_\#10 in LVN network ID_\#12 when this vehicle has arrived the area of SAP ID_\#10 M LVN network_\#12. The SAP ID_\#10 sends immediately this relationship information to the CSMA[7]. And the CSMA modifies the switching-mapping table using this rêlationship of vehicles and LVN network accessed.

The mode bit(2-bit: bit 27 and 26) indicates the direction of vehicle's driving, and that is important index to decide the selection of SAP to provide a smooth handover. The criticalposition bit(2-bit: bit 25 and 24) indicates the start time of handover procedure. The handover procedure is starting when the bit is 01, the CSMA sends one-successive stream to two/or three SAPs(neighbor SAPs) at the same time in accordance with the position of vehicles serviced. The critical-position bit is sent by vehicle to the CSMA through SAP accessed. The critical-position is calculated by GPS system in vehicle, and its position is overlapped coverage area of the SAPs of forwarding direction.

The structure of switching-mapping table on the CSMA is shown in Figure 4, and that is similar to that of paper 10. The first row of unicast mapping table indicates that the vehicle with virtual IP_\#1010H entered into LVN net_ID_\#0010B for the first time in VCN network and accessed SAP ID_\#0010B, and the next that vehicle has driven into LVN net ID_\#0110B 
and accessed SAP ID_\#0001B. The second row of unicast mapping table indicates that the vehicle with virtual IP_\#0110H entered into LVN net ID_\#0101B for the first time in VCN network and accessed SAP ID_\#0100B, and this vehicle is locating on LVN net ID_\#0101B. Thus, the CSMA switches successive streams from LVN net ID_\#0010B and SAP ID_\#0010B to LVN net ID_\#0110B and SAP ID_\#0001B based on the first row of this switching_mapping table. And the third row indicates that the CSMA switches successive streams from LVN net ID_\#0010B and SAP ID_\#0101B to LVN net ID_\#1010B and SAP ID_\#0010B. Of course, the CSMA does not switch the successive streams in the case of the second row. This mechanism can provide a seamless service because the CSMA switches the successive streams based on the mapping table.

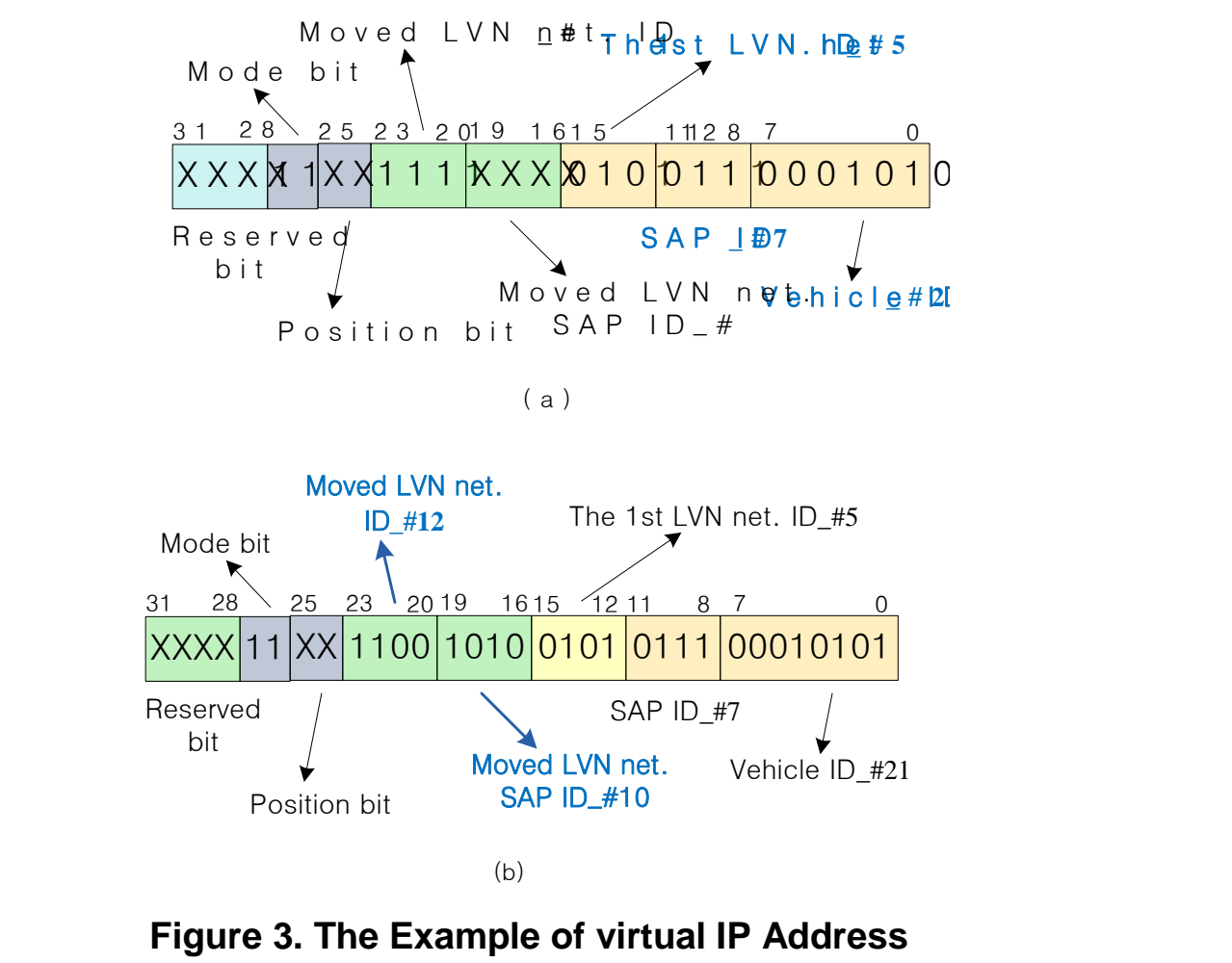

The first row of mullicast mapping table indicates that the vehiclei with virtual IP_\#1110H entered into LVN net ID_\#0011B for the first time in VCN network and accessed SAP ID_\#0010B, and the next that the vehiclei has driven into LVN net ID_\#0111B and accessed SAP ID_\#0100B. The CSMA transmits multimedia streams with MGA ad-dress_\#0101 when the vehicle enters into LVN net ID_\#0011B.

The position bit(2-bit-long) indicates vehicle's position that is the critical one of the next SAP accessible while driving and this procedure uses GPS information. The driving vehicle signals to CSMA to set these bit. The CSMA received this packet sends the same streams to two/or three SAPs(neighbor SAPs) at the same time.

\section{Algorithm for the Multimedia System in VCN Network}

This section presents two algorithms for the proposed mechanism in VCN network. One is the service algorithm about switching streams transmitted from multimedia 
server and management of mapping table using CSMA, the other is handover algorithm that provides smooth handover for driving vehicles in VCN network.

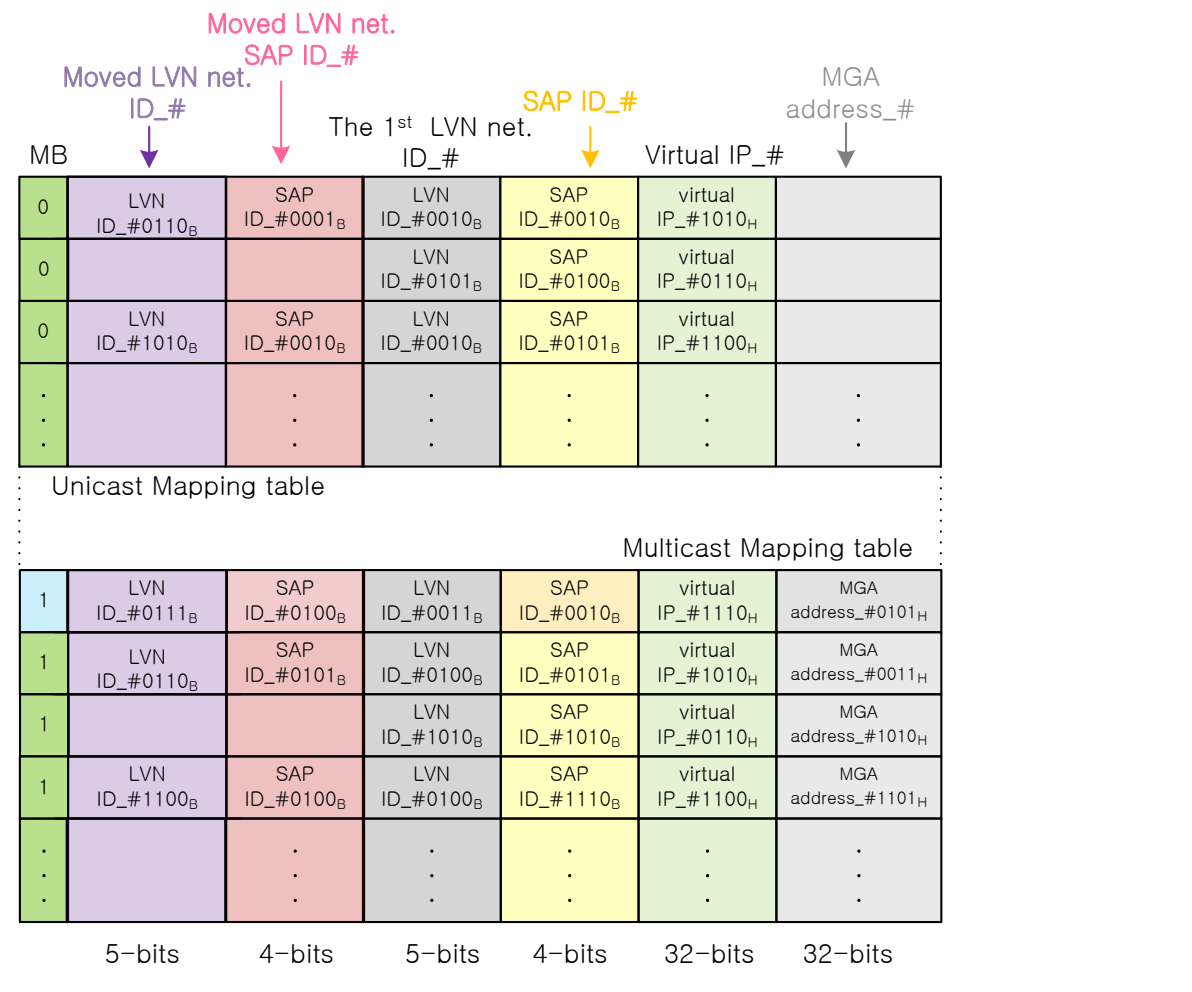

Figure 4. The Structure of Switching-Mapping Table on the CSMA

\section{Service algorithm for the proposed mechanism in Vehicle Communication} Network

i) The yenicle $i$ enters $y C N$ network for the first time, and then accesses $S A P_{-} i$ 13 with vehicle speed

ii) The SAP_i assigns $E V N$ net ID_\#i and SAP_\#i as IP address( IP_\#i) to the vehicle $i$

iii) The vehicle $\left(1\right.$ requests multimedia service_\#i to server through the $S A P \_i$ and CSMA with driving distance using GPS information

iv) The CSMA checks mapping table whether the 1st segment of video_i is stored on buffer. And it checks whether the video requested is live-video If the video requested is live-video, then it generates MGA and the vehicles requested live-video join $M G A$.

v) The CSMA records the IP_\# of vehicle_i and video_ID on mapping table

vi) If the 1st segment of video_i is stored on buffer, then the CSMA transmits it to the SAP_ifor vehicle_ $i$

vii) The CSMA requests consecutive streams of video_\#i to the video server

viii) The video server transmits video streams to the CSMA, and the CSMA delivers them to the $S A P \_i$ 
ix) The CSMA checks whether the transmitting stream is the last one If that is the last stream, then it cut the connection for the vehicle_ $i$ else the CSMA delivers consecutive streams

x) The vehicle_i checks the driving distance whether it arrives critical point If it arrives that point, then the vehicle_i sends its modified IP address to the CSMA through the SAP_i and jump to the Handover Procedure else goto step ix)

\section{Handover algorithm for the proposed mechanism in Vehicle Communication Network}

i) The CSMA checks whether it receives the packet from the step $x$ ) of the Service

ii) When the CSMA received the modified IP address of vehicle_i including the critical point, then the CSMA transmits consecutive only one packet to the SAP_i and neighbor SAPS of the direction of vehicles heading due to the modebit simultaneously

iii) The neighbor SAP(SAP-j) stores the packet transmitted from the CSMA in step ii)

iv) The vehicle_i attempts accessing to the neighbor SAR

v) The SAP_j assigns a new SAP_number(SAP_\#) and moved LVN net ID to the accessing vehicle_i

vi) The vehicle_i decides whetheril changes the ID information of vehicle_ $i$

vii) When the vehicle_i receves $S A P$ jas a new $S A P \_n u m b e r$ from $S A P \_j$, the vehicle_ $i$ necks whether the last packet transmitted is lost or errored.

At the same time the SAP_j sends anew IP of the vehicle_i to the CSMA

viii) The SAP j records the vehicle $i$ entered its boundary to its managing table

ix) The CSMA records a new ID information of vehicle_i on mapping table and it checks whether the last packet transmitted from the previous step(step ii) has occurred problem

If that packet has no problem, then the CSMA transmits the consecutive streams to the SAP_j only and other neighbor SAPs in step iii remove that packet - else the SAP_j sends that stream stored from its buffer to the vehicle_ $i$

$x$ ) The SAP 7 removes the stream(from step ii) from its cache when it is not the $1^{\text {st }}$ video stream of requested video

xi) Enally, jump to the step (vii) of the Service Algorithm procedure.

\section{Analysis of Simulation for the Proposed Multimedia System in VCN Network}

Performing simulation for the proposed algorithms in vehicle communication network(VCN), the simulation environment is that VCN network consists of a CSMA, 25 SAPs and a number of vehicles. 
The simulation for the proposed system is performed that the speeds of vehicles on the road with SAPs in VCN network are $20 \mathrm{~km} / \mathrm{h}, 30 \mathrm{~km} / \mathrm{h}, 60 \mathrm{~km} / \mathrm{h}$ and $100 \mathrm{~km} / \mathrm{h}$ and the driving time of vehicle is 15 minutes. And the reason that adopting the speed of vehicles using $20 \mathrm{~km} / \mathrm{h}, 60 \mathrm{~km} / \mathrm{h}$ and $100 \mathrm{~km} / \mathrm{h}$ is to apply various road conditions. The speed of vehicles is 20 $\sim 30 \mathrm{~km} / \mathrm{h}$ that means the case of congested road condition, $60 \mathrm{~km} / \mathrm{h}$ is normal road condition and $100 \mathrm{~km} / \mathrm{h}$ is the case of highway road. The distance of road of VCN network is $5 \mathrm{~km}$. And the numbers of SAPs in VCN network are 25 that have the radius(100m) of transmission coverage of SAP.

The Figure 5 shows the results of the performance of the proposed mechanism. It shows the comparison of data amount transmitted from CSMA and the number of switching of 25SAPs according to the speed of vehicles in VCN network. Of two-axis in accordance with the speed of vehicles, the left-axis indicates the number of switching of SAPs(SAP\#1, SAP\#5, SAP\#10, SAP\#15, SAP\#20 and SAP\#25) and the right-axis indicates of the amount of data from CSMA. As shown in Figure 5, the amount of data from CSMA decreases according to the increase of the speed of vehicles in VCN network. The reason of decrease the amount of data transmitted from CSMA is that the number of vehicles is more decrease the faster of vehicles on the road in $\mathrm{VCN}$ network.

And the reason of increase the number of switching on the SAPs ac-cording to the speed of vehicles $(20 \mathrm{~km} / \mathrm{h}$ to $30 \mathrm{~km} / \mathrm{h})$ is that the number of vehicles is more $\mathrm{nn} 30 \mathrm{~km} / \mathrm{h}$ including passing by the road in VCN network.

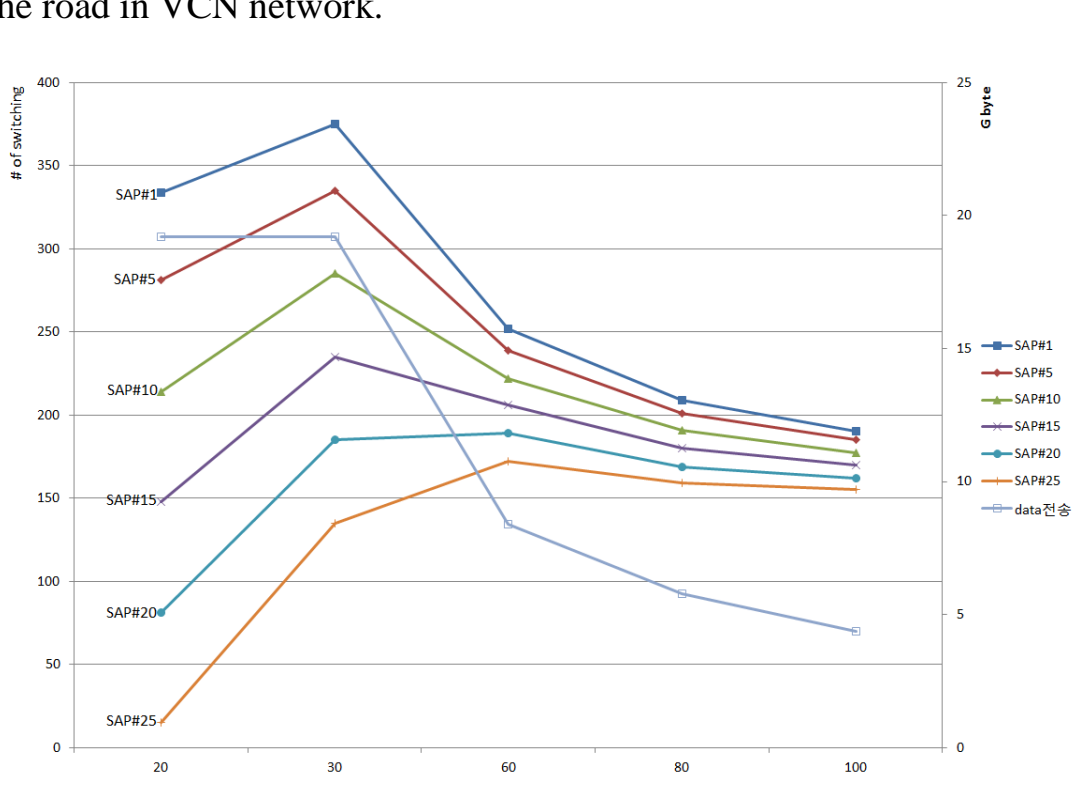

Figure 5. The Result of Performance for the Proposed Mechanism

\section{Conchusion}

This paper proposes a seamless multimedia service system using a CSMA, a number of smart access pointer(SAP) and a partial GPS information in vehicle communication network(VCN). It can provide smooth handover in VCN network using CSMA that manages its mapping table for driving vehicles and SAPs. Also, it can reduce packet loss/error because the CSMA sends the same packet to neighbor SAPs during handover. This mechanism uses a new virtual IP address that is composed of five fields (32-bits long), and the virtual IP address is shorter than the previous paper. And a new research on traffic alerts for safety using this proposed mechanism in VCN network is in progress. 


\section{Acknowledgements}

This work was supported by the Incheon National University Research Grant in 2014.

\section{References}

[1] M. Jerbi, S. Senouci, Y. Ghamri-Doudane and M. Cherif, "Vehicular Communications Networks: Current Trends and Challenges", Handbook of Research on Next Generation Networks and Ubiquitous Computing, IGI-Global, (2009).

[2] G. Karagiannis, O. Altintas, E. Ekici, G.J., Heijenk, B. Jarupan, K. Lin and T. Weil, "Vehicular networking: A survey and tutorial on requirements, architectures, challenges, standards and solutions", IEEE Communications Surveys \& Tutorials, ISSN 1553-877X, vol. 13, no. 4, (2011), pp. 584-616.

[3] I. Kim, B. Kim and Y. Woo, "VOD Service using Web-Caching Technique on the Head-End Network", ICCSA 2003, Springer Verlag LNCS 2668.

[4] G. Gehlen, E. Weiss and B. Walke, "Architecture of a vehicle communication gateway for miedia independent handover", Proc. of the 3rd Int'l workshop on intelligent transportation, Hamburg Germany, (2006).

[5] J. Choi, Y. Woo and I. Kim, "Vehicle-to-Infrastructured(V2I) Multicast Transmission for Live-Video Streams”, IST2013 conference, Bali, Indonesia, (2013) June 27-30.

[6] U. Dwivedi, A. Rajawati and A. Upadhyay, "Characterize an approach to improye performance of multimedia services over vehicular ad-hoc network", Information System and Communication. ISSN: 09768742 \& E-ISSN: 0976-8750, vol. 3, (2012).

[7] Y. Woo and I. Kim, "Vehicle-Based Multicast Communication System for Live-Video", International Journal of Multimedia and Ubiquitous Engineering, vol 8, no. 5, (2013), pp. 325-332.
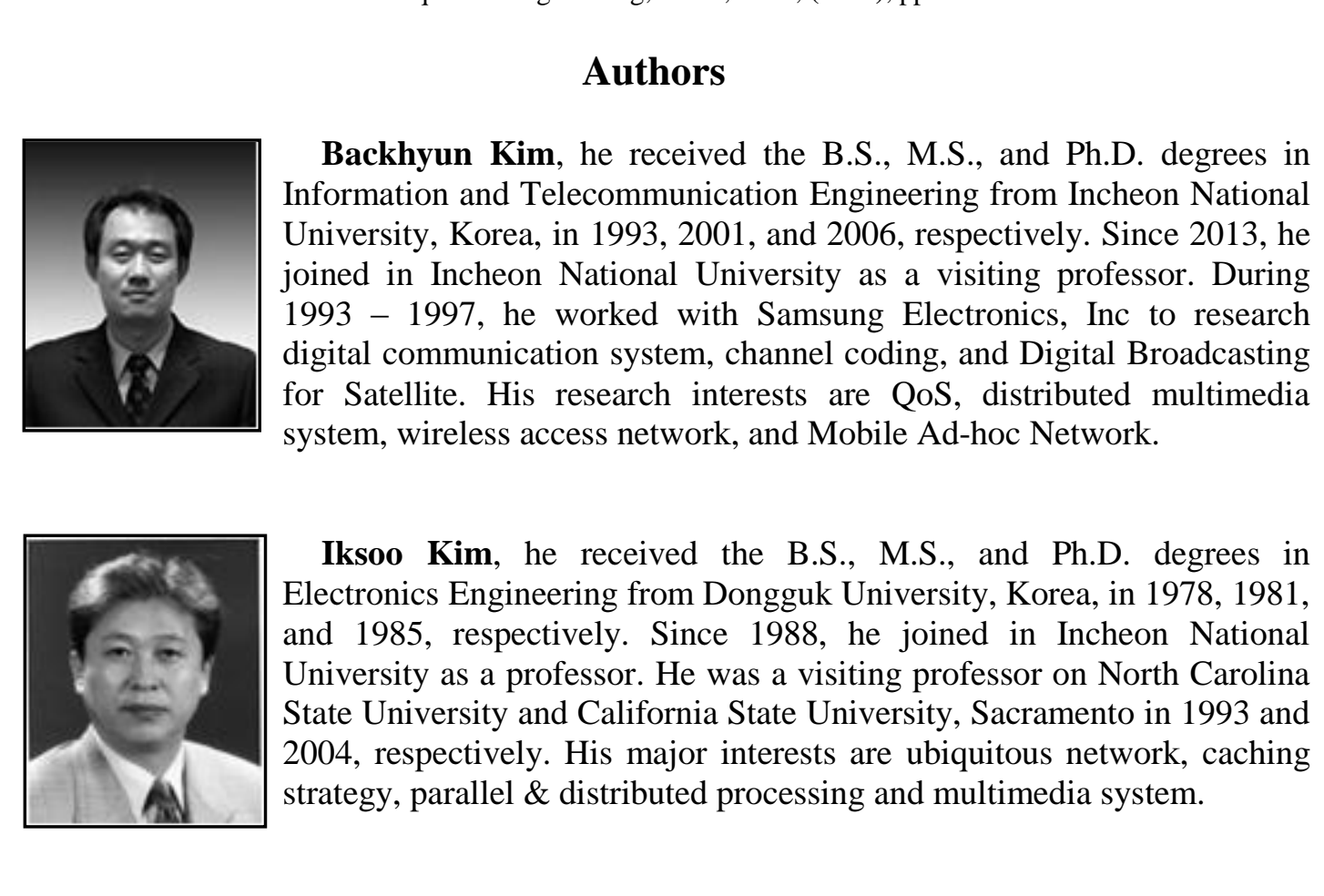

Iksoo Kim, he received the B.S., M.S., and Ph.D. degrees in Electronics Engineering from Dongguk University, Korea, in 1978, 1981, and 1985, respectively. Since 1988, he joined in Incheon National University as a professor. He was a visiting professor on North Carolina State University and California State University, Sacramento in 1993 and 2004, respectively. His major interests are ubiquitous network, caching strategy, parallel \& distributed processing and multimedia system. 
International Journal of Multimedia and Ubiquitous Engineering Vol. 9, No. 11 (2014)

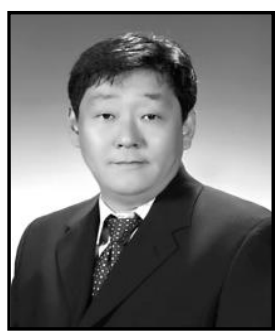

\section{Yoseop Woo}

February, 1992 : A Ph.D. degrees in Electronics Engineering from Hanyang University, Korea.

The present : A professor in department of Information and telecommunication engineering University of Incheon

Major area of interest : Data processing Korean language, Network System, Database.

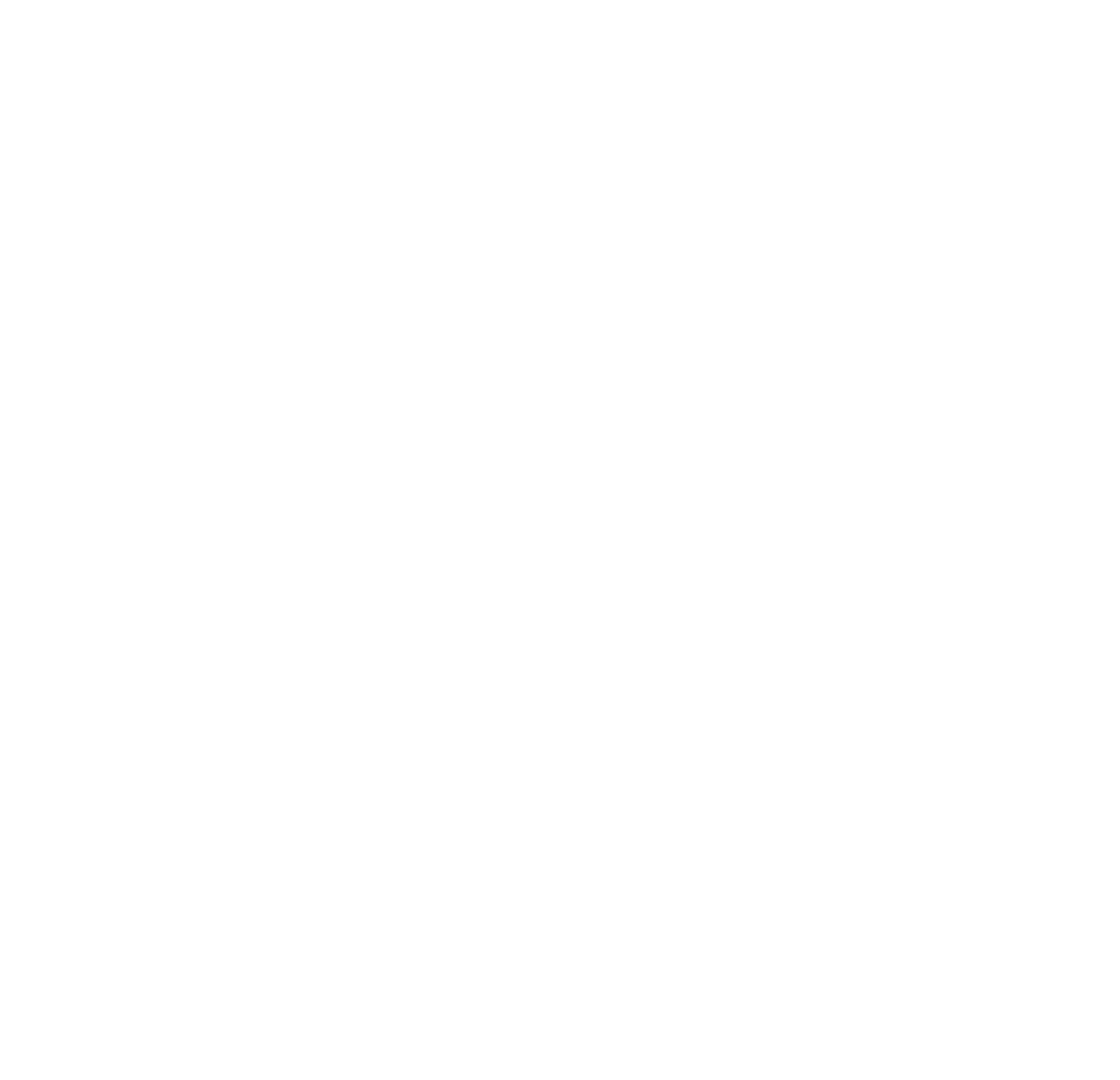

